posing rather than an exciting cause. I have generally found that the cases of variola which were induced by inoculation have been milder than others; and this may account for the facility with which this patient was cured. I conclude that this may be attributed to the absence of any marked constitutional debility.

CASE III. Goolab, aged 25, became an out-patient May 17th 1856 , with variolous corneitis. The disease commenced twelve days after the separation of the crusts. The same remarks are here applicable with regard to the interval of time between the variolous outbreak and the invasion of the corneitis.

CASE IV. Ramjaun Ali, aged 22, became an out-patient June 6th, 18t6, on account of corneitis and its results-dense opacities of the cornea. The inflammation had commenced fourteen days after the invasion of the fever. The opacities were in part removed.

REMarks. In this case, the interval of time was not as great, but the local affection seems to have been of a mild character; for I was only called upon to treat the consequences of the corneitis, and the case was in progress when the patient leit off attending. These results were not indelible.

CASE v. Onrajee, aged 9, became an out-patient on June 9th, 1856, having chronic abscess of the cornea. Three months previously, an attack of variola came on, not from inoculation. The friends said that, at this time there was a small-pox pustule on the surface of the eye, and that the crust from this became separated at the same time as from the pustules on other parts of the body. Inflammation of the eye came on ten days after this separation. The treatment consisted of local depletion, purgatives, hydrargyrum cum cretâ, etc.

June 23rd. 'The mouth had become slightly affected; but the patient was improving, and the matter of the abscess was becoming absorbed.

July 2:3rd. The abscess was healed. The opacities of the cornea in its vicinity were thinning. The patient was in process of being cured.

REMARKS. The corneitis does not seem here to have been part of the external manifestation of the variola, but to have occurred subsequently. The pustules, said to have been in coexistence with the small-pox in the body, most probably only affected the eyelids.

CaSE vi. Banepur, aged 10, was admitted as an out-patien on July 14th, 1856. Variolous ophthalmia was present in both eyes. In the right, in which the attack was of a primary nature, the cornea had sloughed; in the left, the disease hai appeared secondarily - the effect being extensive ulceration and opacities of the cornea. The attack of small-pox had occurred six months previously.

REMARKS. This case is particularly instructive; as, some time after the invasion of the primary eruption, which was attended by so much local disease that one cornea was destroyed by sloughing, another distinct disease, involving also specially the cornea in the other eye, was set up. Nothing seems more probable than that the primary affection was a variolous pustule, having its seat in the conjunctiva and cornea, running a rapid course, and producing mortification of those parts in the same way that variolous pustules act in other parts of the body; and that after a certain interval of time, when the local affection had subsided, the constitutional taint predisposed to the secondary inflammation which seems in such cases to have a tendency to attack some of the vital parts of the organ, which had escaped the more dangerous primary disease. This secondary affection being altogether milder than the other; in this instance, there is every reason for thinking that the disease was perfectly curable.

General Remarks. The cases above noticed afford good instances of the particular forms of the corneitis, which owes its origin to variola. In almost all, the disease showed itself in the most severe form, the one which rapidly runs into destructive ulceration of the structure, instead of into the granulo-vascular degeneration. This proneness to a destructive form of inflammation would be easily accounted for if we could look upon the disease as simply a variolous pustule formed upon the membrane, as in other parts of the surface of the body, but the history of the case will seldom permit us to look upon the case as being one of this description. Indeed, it has been doubted whether the specific variolous pustule is ever formed on the surface of the body, by observers who have had so much experience in small-pox as $\mathrm{Dr}$. Gregory and $\mathrm{Mr}$. Marson, who say that the phlyctenula or pustule that is found to arise in the consecutive stages of variola, are identical with those which arise in common catairhal or scrofulo-catarrhal conjunctivitis (see Wharton Jones's second edition, page 163).
However, I have seen several cases in which a pustule or ulcer was formed upon the conjunctiva or cornea, ultimately affecting both structures at the same time with variolous pustules on other parts of the face and body. In such cases the pustule has been much more rapidly destructive in its effects than where it has occurred in the succeeding stages of the small-pox, and has in fact produced the same changes, leading to mortification of the subjacent parts, as the variolous pustule does upon the cutis. There seems to be no reason why the specific pustule should not occur as well upon th conjunctiva, which possesses a combination of characters of the skin and mucous membrane, as upon the skin of contiguous and continuous structures. The last of the cases given above seems to confirm this view, for one eye had been lost by a destructive affection of the cornea, which had occurred contemporaneously with the pustules on the body; the other eye being only secondarily affected with an inflammation which was much milder in character than the disease which had caused mortification of the cornea in the first eye. Nothing seems more probable than that in this case we had an exemplification of the two kinds of variolous affections of the cornea; in the one the specific pustule leading to destruction of the part implicated; in the other, the milder form of sympathetic secondary corneitis having its origin in a predisposition caused by the disease having taken effect upon the constitution. At the same time, the fact of only one case in six re ported having the appearance of being an instance of specific variolous pustule of the eye, indicates that such a case must be rather rare.

With regard to the period after the accession of an attack of variola, at which the secondary affection, which ushers in corneitis and ulcer of the cornea, comes on, it seems to be subject to considerable variations. Some authors say that it is about the eleventh or twelfth day from the first appearance of the eruption, and when the secondary fever has commenced, (see W. Jones, second edition, page 162); but in the six cases above noted the time varied from the tenth to the twentysecond day after the appearance of the eruption, and the average was eighteen days, so that when this affection occurs secondarily, in most cases probably it is on account of not so much the secondary fever, as of the dyscrasia produced by the disease.

24, George Street, Innover Square.

\section{Griatmal Commmitations.}

\section{ON THE TREATMENT OF CONTRACTED SO INTS.}

By Holmes Coote, F.R.C.S., Assistant-Surgeon to St. Bartholomew's Hospital, and to the Royal Orthopædic Hospital.

[Read before the Royal Medical and Chirurgical Socicty, May 11th, 1858.]

I BEG to call the attention of the Society to the Treatment of Contracted Joints; and, in so doing, I shall limit my remarks to the knee, although the principle of treatment must be the same in every articulation of the body. But, in the first place, I must assume the following propositions as granted.

1. Diseases of joints, whether acute or chronic, may be almost invariably referred to that state of constitution called struma in the young, and to rheumatism or gout in the adult.

2 . The progress of the disease is always painful and tedious, but no morbid changes occur in any of the tissues by their nature irreparable. The synovial membrane may be thickened, but that is the first stage leading to its absorption; the articular cartilage may be removed, but the extremities of the bones become covered with other tissues; ligaments, softened and elongated, will in course of time become firm, and will even recontract, if put under proper and favourable conditions.

3. The severer changes with which we meet in practice are mainly due to want of proper maintenance of repose of the joint during the course of the disease. Where do we notice in. stances of inflammation of the cancellous texture of the bone leading to abscess? At the lower extremity of the femur, at the upper and the lower extremities of the tibia, but not in the bones of the upper extremity. These accidents happen, then. just to those bones which support the weight of the body. 
and which have that weight thrown upon them when unfit to bear it.

4. If we except malignant disease-i.e, the different varieties of cancer-there is no disease which does not run through its course to cure.

5. Inasmuch as articulating surfaces are, with scarcely an exception, pressed firmly together in the extended position, and held loosely together when the limb is bent, in consequence of the direction and attachments of ligaments and fibrous bands, it follows that a joint, when diseased, is thrown into the flexed position by the patient, as being the easiest possible position.

6. That position may, after a time, become permanent, in consequence of the contraction of tendons, the formation of adhesions, or some displacement of the bones.

7. No attempt at extension and readjustment of the bones of a diseased joint should be attempted, until all active disease is passed, and the patient is free from pain.

8. Whatever may be the course adopted, it is necessary to preserve the continuity of the surrounding tendons, that the extended limb may be under the control and direction of the patient.

I do not hesitate to avow my belief that, in the treatment of diseased joints, there has not hitherto been shown, generally speaking, sufficient patience on the part of the surgeon, combined with the strict maintenance of rest and proper support of the affected limb. An idea has prevailed that certain morbid changes demand amputation; and this measure has been adopted upon very insufficient evidence as regards the incurability of the affection. Less patience and pathological research still has been shown by those most enthusiastic in the cause of excision of the articular extremities of bones; and it is with a feeling akin to horror that I read of the performance of the operation upon infants of six years of age. Out of thirty recorded cases of excision of the knee-joint, severiteen of the patients were between six and sixteen years of age-in fact, just at that period of life when best able to surmount the exhausting influence of disease.

At the risk of being accused of insisting upon that which is generally acknowledged, but which, I must add, if acknowledged, is not acted upon, I will briefly relate some cases to illustrate the progress of three forms of disease; namely-

I. Common strumous enlargement.

II. Light brown pulpy degeneration of the synovial mem. brane.

IIr. Rheumatic inflammation.

The cases which I will select will show not only how readily the process of repair goes on, if nothing is done to disturb its course, but likewise the frequent haste with which a joint is condemned; for, in nearly every case to which I shall allude, some severe operation had been recommended.

The Fellows of this Society will, I am sure, appreciate my motive in withholding names in the histories of the cases, and will accept my guarantee that I state that which is supported by proper evidence.

I. Strumous Disease. I was requested on February 28th to give my opinion, in consultation, upon the following case. A little girl, aged $\tau$, had suffered for two years from strumous enlargement of the right knee. The leg was drawn backwards into the ham, and rotated outwards by the action of the biceps; the limb was semiflex. Attempts at the extension of the joint by force had been tried, and had failed, although the hamstring tendons had been divided. The knee was hot and swollen. It was urged that, inasmuch as the tibia was displaced from the lower extremity of the femur, the limb could no longer be of use; and amputation was proposed. Upon my dissenting from such a course, the case was put into my hands I bandaged the limb from the toes upwards, and applied to the popliteal space one of the padded splints now before you, bent to suit the angle of the linee. A poultice was put upon the joint, until the heat had passed away; and then gentle pressure was kept up by means of the splint; and the knee is becoming daily straighter. I have no hesitation in saying that, in the course of a few years, there will be no trace (with proper precaucions) of the present disease; but that a few weeks will suffice to enable the patient to leave her bed and walk with a stick.

Now, one of the surgeons who examined the case doubted the possibility of the replacement of the bones, and the future usefulness of the limb; and he accordingly accompanied me to a ward in St. Bartholomew's Hospital, where he saw the following case.
Mary Anne H., aged 22, sprained her left knee when quite a child, in Galway. The limb swelled and became painful ; and the surgeon who attended her advised amputation. The parents would not consent, and ultimately she was able to creep about. For sixteen years she has walked upon crutches.

She was admitted under my care December 18th, 1857. The tibia and fibula of the left leg were rotated outwards, and drawn backwards, the leg being semiflexed, into the popliteal space. The lower end of the femur projected, supporting the patella: these were the remains of a fistulous passage quite healed. The toes were rather more than six inches from the ground. I divided the tendons of the biceps; and, having kept the divided ends in apposition for five days, I applied moderate extension by means of a weight (seven pounds) attached through a pulley to the foot. Immediate pressure to the displaced bone was made by splints.

February 24th. She walked, without crutches or other aid, from the ward; the foot having been brought four inches and a quarter nearer the ground. The bones of the leg had been partially brought under the femur; and she had a shoe with a high sole. Had I had at disposal such means as are to be obtained in private practice, I could have obtained a full half-inch more of length.

II. Pulpy Thickening of the Synovial Membrane. In this form of disease, the synovial membrane becomes converted into a thick, soft, light brown coloured mass. The microscope shows that it consists of a multitude of granular corpuscles, which are acted upon by the absorbents; for the same mate. rials are found in the neighbouring absorbent glands. The disease has been so well described by Sir B. Brodie, and is of so frequent occurrence, that no further description from me is necessary.

In opposition to the opinion of some of the most respected of my professional brethren, I am positive that this disease is not incurable. It constitutes a stage in articular disease, of which the changes are, absorption of the synovial membrane; absorption of cartilage; fibrous union between the denuded bones. In the summer of 1854 , a young woman was in St. Bartholomew's Hospital, under my care, with this condition of the synovial membrane in the right knee. No operation was ever seriously contemplated; but she would not listen even to an approach to the question. In the course of a few weeks, the pain and swelling subsided, and she quitted the Hospital. I have seen her often since that time, and, with care, she manages to get about very fairly.

Now, what does pathological investigation show? In 1846, I examined a knee-joint amputated for this affection of the synvovial mernbrane. The synovial membrane was thickened, softened, and discoloured, and overlapped the articular cartilage, which was loosened from the subjacent bone. The bone was vascular, but otherwise healthy. In 1850, I examined a knee-joint in an amputated $\operatorname{limb}$, with the following re ult. The knee-joint was divided into three compartments, in consequence of adhesions between the remains of the synovial membranes : one between the outer condyle of the femur and the corresponding surface of the tibia; another between the inne: condyle of the femur and the corresponding surface of the tibia; the third between the patella and the outer condyle of the femur. The cartilages were removed by absorption. Now, this joint had not been kept quiet during the active stage of the disease. The surfaces of the bones were rough and grooved, and locked into one another, the groove correspond. ing with the line of movement. The bone had also in parts inflamed, and become necrosed. In 1848, I examined a lneejoint, also removed by amputation for pain of the part, in which it was found that the tibia, fibula, and femur, were quite healthy, and united by fibrous membrane, the synovial mem. brane and the cartilage having been absorbed.

I believe that, in both forms of disease to which $I$ have alluded, the changes consist in the gradual removal of the proper tissues of a joint, and of the union of the denuded bones by fibrous tissue. Bony anchylosis is of the rarest possible occurrence.

The idea of incurability should be dismissed; neither amputation nor resection should be thought of. The limb should be kept quiet until the morbid changes have come to an end; and then the contracted limb should be slowly extended.

IIr. Rheumatic Disease. Rheumatic disease is either acute or chronic, and the former is often very severe; but I doubt in the frequency of well managed cases requiring amputation. Last September, a young man, a sailor, was admitted into St. Bartholomew's Hospital, with acute rheumatic inflammation of the left hip. So severe were his sufferings, that I am sure 
amputation would have been recommended, had the knee-joint been the seat of the disease. The cartilages were absorbed, and the head of the femur ulcerated, so that the limb was shortened and everted. But, after a time, the pain subsided, and the limb was brought down; and he quitted the hospitalon a crutch, it is true; but experience shows us that ultimately he will be able to bear upon the limb.

A young girl is at the present moment under the care of my colleague Mr. Tamplin, at the Royal Orthopædic Hospital. She suffered in 185:3 from acute rheumatic inflammation of the right knee. Amputation was at one time thought of by the surgeons who attended her, so severely did she suffer. She was admitted September 4th, 1857. The leg was firmly semiflexed on the thigh, but all pain and swelling had subsided. A proper instrument was applied.

January 4 th, 1858 . The leg is so much straighter that she can support herself upon the toe.

February 22nd. The limb is still under treatment. She can put her foot to the ground.

After rheumatic disease, bony anchylosis is not so very uncommon. There is a wide difference in this respect between the consequence of rheumatism and of struma. More force is, therefore, frequently required in the extension of contracted limbs from rheumatic disease; and such a proceeding may even be unadvisable in some situations, such as the carpus. A young woman is now under Mr. Lawrence's care, in St. Bartholomew's Hospital, who, after one attack of rheumatic fever, suffered from contraction of all her joints. The bones of the left wrist appenr united by bony anchylosis.

There are three methods by which we may extend a contracted joint.

The first, namely, that adopted by Louvrier and Dieffenbach, I will brietly notice, as utterly unfit to be practised, owing to the dangerous, violent, and unscientific nature of the proceedings. The bands of alhesion, contracted tendons, and all resisting structures, were forcibly torn asunder; the limb, once straightened, was held so mechanically until the tendency to contraction was overcome. So serious were the results (and some cases ended fatally), that the scheme was soon abandoned, and is now rarely resorted to, except by some surgeons in exceptional cases of impaired movement of the elbow after injury. It is a proceeding I cannot recommend.

The second consists in the subcutaneous division of tendons, followed, at an interval of seven days, by the forcible rupture of adhesions. The limb is then put up in the flexed position, and, after six or seven days, the soft uniting material is slowly elongated by a proper apparatus. This plan is recommended in some cases by Mr. Brodhurst. (Medico-Chirurgical Transactions, vol. xl.)

The third consists in the slow elongation of the contracted tendons and uniting bands, without forcible rupture, but with or without the subcutaneous division of tendons. This plan is the one usually adopted at the Orthopedic Hospital, and is recommended by Mr. Tamplin. As plan No. 2 has been already well described in the Transactions of this Society, I shall confine my remarks to the third plan.

But, before mentioning some cases by which the particulars of the proceeding may be illustrated, I would call attention to a course which unhappily combines the evil of the first and second plan, without any of the attendant advantages. Some surgeons have first subcutaneously divided the tendons; next they have immediately extended the joint by force, the patient being under the influence of chloroform; thirdly, they have put the extended limb into an immovable apparatus, as if they were dealing with unorganised parts. One moment's reflection would show that, independently of the uncertain amount of injury inflicted, by which dormant disease may be roused into activity, the ends of the divided tendons are widely torn asunder, and cannot be expected to reunite so as to afford the proper support to the joint; while such fibrous bands as, by their position, admit of reunion, will again be liable to recontract, inasmuch as the newly deposited material has all the properties of a cicatrix.

That which is wanted is extension-not laceration; and, if we divide a tendon, we carefully keep the divided ends in apposition, until a new and yielding substance has been effused between them. 'This was first pointed out by Delpech.

Fibrous alhesions admit of great elongation under slowly applied pressure; and such pressure causes the absorption of the contracted element, so that the elongation is permanent.

The careful examination of a contracted joint is necessary, in order that we may form a just prognosis, and determine upon the exact nature of the apparatus and method of treat- ment to be adopted. In the first place, we must ascertain whether there is motion, and, if so, what is the amount; in the next place, the nature of the obstruction, or the cause of resistance; thirdly, we must ascertain whether the patient possesses any voluntary power over the limb.

"After these preliminary inquiries," remarks Mr. Tamplin, "let an assistant hold the thigh firmly, while you attempt to extend the leg forcibly. If you are able to extend it in the slightest degree, and the patient complains of pain on the anterior surface of the joint, however slight the motion obtained in this way may be, your prognosis will be favourable; and, even in those cases where there is no perceptible motion, if the patient complains of pain in the situation mentioned, you may anticipate the possibility of removing the contraction. It is also as well to examine the patella, and ascertain whether it admits of motion in a lateral direction." (On Deformitics, p. 159.)

The character of the motion is then to be ascertained. "If it is a steady motion, and if, upon an increase of extension, the flexor tendons are rendered more tense, you will regard the resistance as confined to the muscles principally; if, on the other hand, you find, upon keeping up the extension for some seconds, that the joint returns to the contracted position with an elastic impulse, you may anticipate resistance arising from adhesions in the joint itself. If you find free motion beyond the contracted or flexed position-that is, if you are enabled to increase the amount of flexion without difficulty, with as much ease as can be done ordinarily - this will be a favourable circumstance." (Op. cit.)

But if a joint be immovable, and occasions no pain upon examination, and if, upon continuing extension, the muscles relax themselves, it is hopeless to attempt restoration by ordinary methods.

The instruments here exhibited have been constructed by $\mathrm{Mr}$. Ferguson of Giltspur Street, and are well worth an atten tive examination. It will be observed that, in place of the old male and female screw at the back of the splint, there are screws at the side which may be so arranged as to give not only the movement of flexion and extension, but likewise abduction, adduction, and rotation. Thus the leg drawn backwards into the ham, rotated outwards, and bent, may be slowly extended, may be rotated inwards, and pushed forwards under the extremity of the femur, pressure upon any sensitive spot being relieved by some combination of action.

The apparatus is very commonly adapted to a boot by which the proper position of the foot is maintained. Provision is made for flexion and extension of the ankle.

The use of this instrument requires care and constant supervision. The great secret, whether any tendons be divided or not, consists in the gradual and painless, yet steady and unremitting application of the extending force.

The following cases have been selected out of many to illustrate its use.

Emma R., aged 8, fell on the left knee three years ago at Cheltenham, and, as she says, put her kneecap out.

A year ago she attended the Royal Orthopædic Hospital as out-patient under Mr. Tamplin. The knee was at this time very much contracted. It was treated upon the principles laid down by the late Mr. Scott, and the patient could walk about with a stick. By this treatment some improvement was obtained.

September 6, 1857. She was admitted into the Orthopxdic Hospital. The limb was still much contracted and swollen, but not painful. The usual instrument was applied.

February 26. The knee is of natural size; the leg is almost straight upon the thigh.

Fanny 'T., aged 15, a delicate girl, had for the last six years suffered from inflammation of the lungs. Three years ago she experienced weakness in the right knee, accompanied with cramps; she became a patient in the Northampton Infirmary, where she was treated, and subsequently discharged. The pain still continued, though diminished, and the leg at right angles to the thigh.

February 15. She was admitted into the Royal Orthoprdic Hospital, under Mr. Adams, when the usual instrument was applied.

February 28. The leg is nearly straight; the joint is free from pain.

I have selected these cases out of many, as showing what may be done with common precaution and care; and when I am met with that most unworthy of all arguments-that such a practice is all very well for the rich, but does not answer with the poor, who cannot spare either time or attention, I reply, that to the poor especially is the preservation of a limb 
of paramount importance. Those who thus speak, seem not to know how many months, and sometimes years elapse before an artificial limb can be worn with comfort; how many means of obtaining subsistence are debarred from one so mutilated; I uphold that any length of time is well spent, which gives the patient the chance of escaping this dreadful alternative, from which, as Mr. Hussey of Oxford has shown, not only do many die, but many more never thoroughly recover.

I will conclude by deseribing the way in which the hamstring tendons are divided and treated, when this proceeding becomes necessary in combination with the above.

Let the paijent lie horizontally on his face; an assistant grasps and extends the leg. A small sharp-pointed knife is passed to the inner side of the tendon and beneath it horizontally. As soon as it is fairly under the tendon, turn the sharp edge upwards, and divide from within outwards, i.e., from the popliteal space to the skin. The biceps flexor cruris is usually the most tense, but such is not always the case. Sometimes the outer hamstring tendon alone requires division; sornetimes both outer and inner. In many cases after the division, the tendon's numerous tight bands spring up when the extension is kept up. Care must be taken not to wound the popliteal vessels and nerves, should interference with them be deemed necessairy. Extension generally suffices. The operation being completed, pledgets of lint are put on the wound, and fixed there by adhesive plaster. The limb is supported by a bent turned splint, and rolled from the foot upwards. It is best to allow a week to pass before any attempt at extension is made, because by proceeding too quickly, the parts of puncture may be irritated, and the treatment delayed. The chief danger consists in the division of the peroneal nerve; and such an accident has happened. It occasions the patient considerable alarm, but in the course of time the divided nerve reunites, and sensation and motion are both restored.

\section{N S A N I T Y.}

By Joln Watson, M.D., Southampton.

WE are indebted to the advocates of phrenology for having of late years given an impetus to the study of insanity. It is a question, however, whether this science has not introduced some disadvantages; among which may be placed the attempts that have been made to distribute the different manifestations of this affec.ion to different portions of the brain, in accordance with their supposed subserviency to the different groups of mental faculties; and thus further to complicate a sufficiently difficult subject. I propose in this paper (1) to adduce reasons for rejecting the view first promulgated, I believe, by the late Dr. Prichard, and adopted, under the same or some equiralent name, by most recent writers both at home and abroad-that we can have what is called moral insanity, while the intellectual powers remain unaffected; and (2) to view the entire question from a different stand-point.

1. That insanity can be anything else than an aberration of a man's intellect is a startling proposition. It would be quite as reasonable to separate such cases as exhibit sensory illusions into a distinct class under the head of sensational in. sanity (thus mistaking an accidental condition for the essential disease), as it is to affix the term moral insanity to those cases which exhibit particular excitement or perversion of the moral sentiments. In a proper, restricted sense, indeed, terms expiessive of the cause or course of insanity are in common use, cuch as religious, homicidal, suicidal, puerperal, etc.; but these are merely qualifying expressions, and are not used in contradistiaction to insanity of the intellect. And, in the same way, ihe term moral insanity, meaning the moral causes or moral consequences of insanity, would be unobjectiorable. Etymologically, this phrase means either unsoundness of morals, which is vice, or insane conduct, which is simply an inseparable accompaniment of insanity, and reveals it. But, beyond the objection there is to the phrase itself, the doctrine it represents will not, I think, bear investiration. It is meant that the sentiments, the enotions, as distinguished from the intellect, are insane. Emotion, I submit, may be designated as warm, enthusiastic, cold, depressed, perverted, or non-existent; it may be in excess or deficiency; but to call it insane is meaningless, and, if ever conventionally allowable, cannot be permitted in scientific language. In examining the various sources whence human actions spring, we shall find them reducible to either an instinctive impulse or a motive; the first being a motion of the organism, and the last a conception of the understanding. To which class does moral insanity belong? If to the former, then it is the province of a sound intellect to restrain its irregular instincts and emotions; but if to the latter, then we have insanity, properly so called. But, again, the distinct line that exists in the mind between the operations of the understanding, and the sentiments or emotions, is entirely overlooked by the advocates of this doctrine. Our moral nature is said to consist wholly in these sentiments or emotions; thus forgetting the fact that a moral judgment invariably precedes a moral sentiment, and that they stand to each other in the relation of cause and effect. A little reflection will make this apparent. The sentiments of reverence and gratitude towards God follow a rational conception of his being and attributes; remorse, the judgment that we have acted basely; pity, the concention of suffering; indignation, that of injustice; and so on. "The perception", says Royer-Collard, "of the moral qualities of human actions is accompanied by an emotion of the soul that is called sentiment. It is a fact that, by the contemplation of a beautiful action or a noble character, at the same time that we perceive those qualities of the action and the character (perception, which is a judgment), we feel for a person a love mingled with respect, and sometimes an admiration that is full of tenderness. A bad action, a loose and perfidious character, excite a contrary perception and sentiment." (Cousin's Lectures, by Wight: The True, the Beautiful, and the Good. Lect. 13.)

These considerations point to the conclusion that no real distinction exists between intellectual and moral insanity, and that moral insanity necessarily implies intellectual misconception.

2. Reverting to the general question of insanity, and bearing in mind that any lengthened metaphysical details are unsuited to the pages of a strictly medical journal, I will now attempt to draw an outline of the subject, merely stating results which $I$ assume (if need were) could be fully established.

The world of mind is revealed to each individual in his own consciousness, and an attentive examination of what passes therein will satisfy us that all its phenomena may be grouped, as is ordinarily done, under the three heads-1. Operations of the understanding; 2. Feelings; and 3. Voluntary power. A still more careful examination enables us to divide the operations of the understanding into two great classes-1. Intuition or spontaneity; and 2. Reflection, or the logical faculties. In its intuitional activity, the reason acts by insight, seeing " the truth as the eye does light, only by being directed toward it." (Locke's Essay, bk. iv, ch. 2.) Hence do we derive all universal necessary truths, and the first principles of all knowledge. (See Reid, Sir William Hamilton, and Cousin.) In its reflective activity, reason acts by reasoning (observation, deduction, and induction); and hence do we derive all relational knowledge, and the arts and sciences. The feelings also are divisible into-1. Sentiments or emotions; and 2. Sensations. Sentiment is defined as a sensible response excited by a conception of the understanding. Sensation, on the other hand, is excited by or through a bodily organ, and may be either an instinct or the action of one of the special senses. The will is the free power we possess of determining inward or outward activity.

As giving distinctness to what has just been written, I may be allowed to reproduce it thus :-

1. Understanding :

\section{CONSCIOUSNESS.}

a. Intuitional, or Spontaneity-Reason acting by Insight. Necessary truths: the Infinite: the Good: the Beautiful.

b. Logical, or Reflection-Reason acting by Reasoning. The relations of things.

2. Fceling:

a. Sentiment or Emotion: Love, Faith, Hope, Joy, Aspiration, Sorrow, Remorse, Awe, Veneration, etc.

$b$. Sensation-Instinct: The Special Senses.

3. Will : The Principle of Personality: a free Power of determining inward or outward actions.

Which of these classes of faculties is it, whose disorder that constitutes the essence of insanity? We exclude the feelings, because, as we have seen, they are instinctive tendencies which are susceptible only of differences in intensity, and insanity does not consist of intensified or destroyed feeling; and because this part of our nature is placed under the control of a will directed by a sound understanding. We exclude the will, because perversion of this principle constitutes, not a physical disease, but that moral disorder with which spiritualists and moralists, or legislators, alone have to do; and 